\title{
flexiơn esviada: estado actual del tema
}

F. MORAN y A. VERDE, ingenieros de caminos

451-4

Se presentan brevemente las diversas posibilidades existentes para el dimensionamiento práctico de secciones de hormigón armado solicitadas en flexocompresión esviada, en el marco del cálculo en rotura.

\section{Introduceiøn}

La comprobación de una sección de hormigón armado solicitada en flexocompresión esviada es un problema encontrado con relativa frecuencia en la práctica, ya que a esta solicitación están sometidas no sólo las secciones simétricas que soportan una fuerza actuando fuera del plano de simetría, sino también todas aquellas que no son simétricas por su forma o por la disposición de sus armaduras. Puede incluso decirse que la flexión esviada es el caso general, del que la flexión recta es una simplificación sólo admisible cuando se trata de secciones simétricas en las que no se consideran los momentos normales al plano de simetría, como son los debidos al pandeo fuera de dicho plano. Si bien es cierto que hasta ahora la gran mayoría de las secciones se calculaban en flexión recta, también lo es que, mientras que para flexión recta existían fórmulas y ábacos de aplicación inmediata universalmente aceptados y difundidos, no era éste el caso de la flexión esviada, en el que era preciso emplear alguna receta para reducirla al caso anterior, con gran quebranto económico y a veces sin garantía de seguridad, o bien abordar la resolución rigurosa, que fácilmente podía consumir horas de trabajo cuando hubiesen bastado minutos.

El Instituto Eduardo Torroja se viene ocupando del tema con vistas, por una parte, a su inclusión en la Instrucción Española de Hormigón E.H. 68 y, por otra, a su puesta a punto en el marco de la Comisión III (Flexión-Compresión) del Comité Europeo del Hormigón. Los autores de este artículo han realizado estos estudios bajo la dirección y con la colaboración de los Dres. ingenieros José Antonio Torroja y Alvaro García Meseguer.

\section{Solución general del problema}

Desde el punto de vista teórico el problema no presenta dificultades, si se aceptan las hipótesis admitidas por el C.E.B. para la flexión recta. En efecto, el agotamiento resistente de la sección viene caracterizado por una serie de posiciones del plano de deformaciones. Para cada una de estas posiciones se conoce la deformación en todos los puntos de la sección; y de los diagramas tensión-deformación de los materiales se deduce la tensión en cada punto de la misma. La integral de estas tensiones puede, por consiguiente, calcularse en magnitud y posición, y será una fuerza biexcéntrica que agota la sección. Basta pues con hallar la fuerza que agota la sección con las excentricidades, datos del problema; y éste queda reducido a comparar esta fuerza con la carga mayorada actuante. 
Pero para ello es necesario el empleo de un proceso iterativo enormemente laborioso, inabordable con los métodos de cálculo tradicionales. El cálculo electrónico es indudablemente una buena alternativa; y si bien hoy en día tiene el gran inconveniente del tiempo de acceso al ordenador, que es del orden de 1 a 3 días para la mayoría de los proyectistas, en lo futuro es probable que llegue a ser la solución definitiva, al reducirse este lapso a minutos gracias a la difusión del tiempo compartido. En el Servicio de Cálculo Electrónico del Instituto Eduardo Torroja existe un programa en explotación, que permite la comprobación de una sección de forma y armaduras cualesquiera; los detalles sobre este programa han sido dados en la ficha E-40, aparecida en el número 206 de la Revista INFORMES DE LA CONSTRUCCIÓN. La reproducción de esta ficha puede verse en la figura 1.

\section{Abacos adimensiomales}

Una primera alternativa a la resolución iterativa rigurosa consiste en la preparación de ábacos adimensionales como el que se ve en la figura 2. Para secciones rectangulares con una cierta disposición de armaduras y calidad de acero, mediante una serie de 8 a 10 ábacos, se consigue un dimensionamiento (o comprobación) muy rápido y exacto. El Instituto ha publicado (1) tres series de ábacos para acero ordinario y armaduras iguales en las esquinas, en las caras opuestas y en las cuatro caras (Revista "Hormigón y Acero», número 78), y ha preparado estas mismas series para acero de $4.200 \mathrm{kp} / \mathrm{cm}^{2}$ de límite elástico; la serie correspondiente a armaduras iguales en las cuatro caras se incluye como ejemplo al final de este artículo. Es necesario tener en cuenta que las bases que han servido para la elaboración de estos ábacos (posiciones del plano de deformaciones que caracterizan el agotamiento y diagramas tensión-deformación de los materiales) no coinciden estrictamente con las adoptadas por la Instrucción Española E.H. 68 (2) ni con las últimas ideas del C.E.B., por lo cual el Instituto Eduardo Torroja espera publicar próximamente nuevas series actualizadas.

Análogos son los ábacos de dimensionamiento adoptados en Alemania, donde Rüsch, Grasser y Linse han preparado series como la recogida en la figura 3, válida para armaduras concentradas en las esquinas. Es interesante observar cómo la ingeniosa disposición "en roseta» aprovecha la doble simetría de la disposición de armaduras elegida, para condensar la serie de ábacos en un único diagrama.

El principal inconveniente de los ábacos adimensionales radica en la necesidad de disponer de la serie correspondiente a la disposición de armaduras elegida. Fácilmente se comprende que, para trabajar rigurosamente, sería necesario un número muy grande de dichas series, pues habría que respetar no sólo la distribución de las barras, sino también los recubrimientos. El trabajo que representa la preparación de cada serie, aun disponiendo de un ordenador electrónico, y la molestia que supone la utilización de un elevado número de ábacos, aconsejan en la práctica utilizar unas pocas disposiciones y reducir las otras a ellas, aun a costa de perder exactitud en la asimilación, siempre que sea del lado de la seguridad.

\section{Nomegramea adimeensional}

Otro inconveniente de los ábacos radica en la necesidad de proceder a una interpolación entre dos de ellos, ya que normalmente el valor de $n$, dato del dimensionamiento, no coincide con los de los ábacos de la serie. Esta interpolación es otra causa de inexactitud, pero sobre todo de molestias si hay que dimensionar o comprobar varias secciones. Por ello, los autores han desarrollado un nomograma que recoge en un solo gráfico todos los ábacos de una serie. El resultado puede verse en la figura 4, que corresponde a la serie de armaduras iguales en las cuatro caras y acero ordinario. Naturalmente que esta condensación también implica una pérdida de aproximación, pero afortunadamente está dentro de límites admisibles: en ninguno de los casos examinados superó el $5 \%$, error comparable al de lectura. 


\section{E.T.E.E. \\ División de Cálculo \\ Csthrenctureas \\ flexocompresión esviada}

\section{objetro:}

Comprobación de una sección de hormigón armado, sometida a una compresión biexcéntrica. La sección puede ser de forma cualquiera y tener cualquier disposición de armaduras. Los diagramas tensión-deformación para acero y hormigón se definen por puntos. Se admite que la sección permanece plana y que el agotamiento resistente se produce al alcanzarse en la esquina más comprimida la deformación límite.

\section{cilentoss:}

Los datos necesarios son:

Sección de hormigón, definida mediante áreas elementales dadas por su superficie y las coordenadas de su centro de gravedad.

Armaduras, dando para cada una el área de acero y las coordenadas.

Diagrama tensión-deformación del hormigón y del acero, minorados.

Deformación límite del hormigón.

Para cada hipótesis de carga: excentricidades y posición inicial de la fibra neutra de deformación.

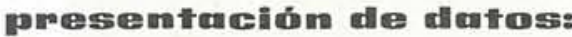

Enviando al I.E.T., División de Cálculo, un croquis en el que figuren todos los necesarios.

\section{mesullintan allos:}

Para cada hipótesis de carga son: posición de la fibra neutra de deformación y valor de la fuerza que agota la sección con las excentricidades dadas.

\section{oloserewcuriomes:}

pille ale puemte

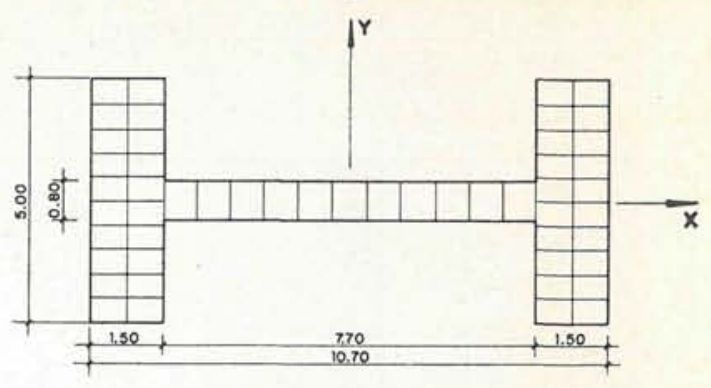

división de la sección en áreas elementales

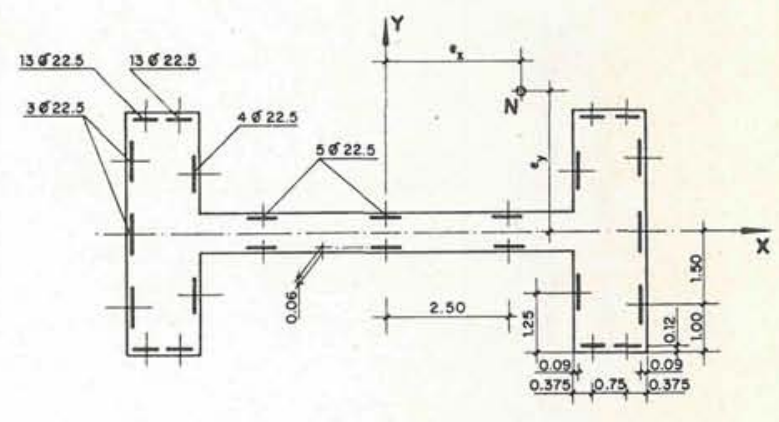

armaduras de la sección y posición de la carga (hip. 2.")

\section{elleatros}

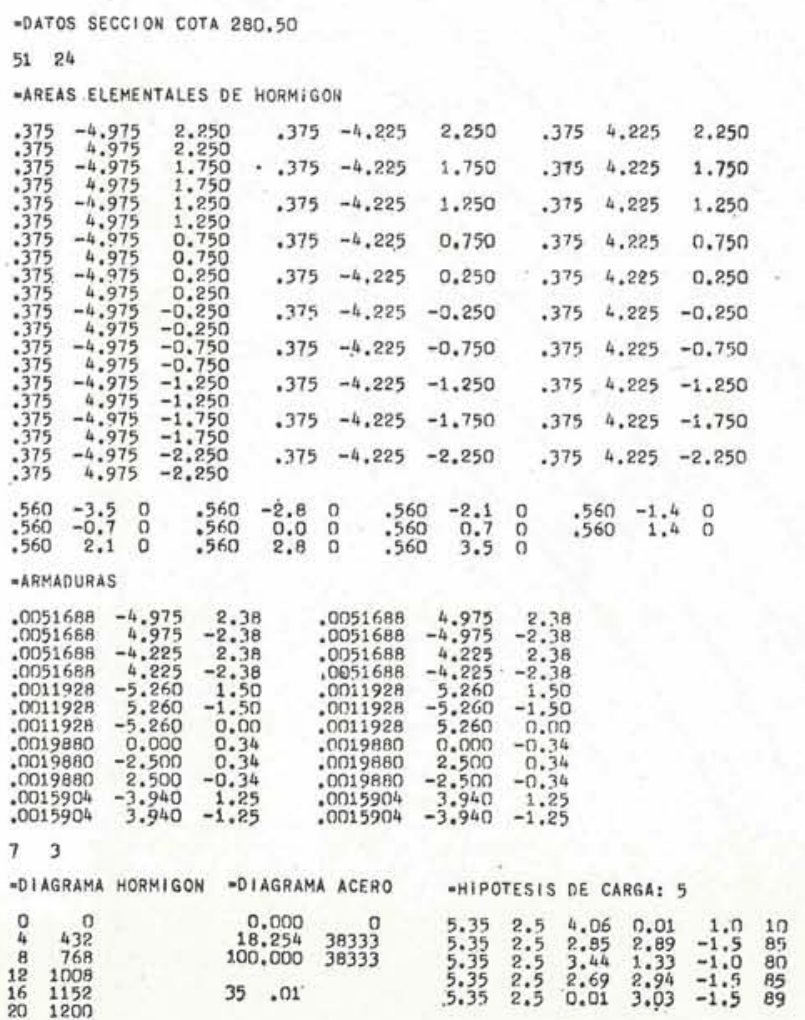

\section{vesulteratos}

RtSULTADOS StCCion COTA 280.54

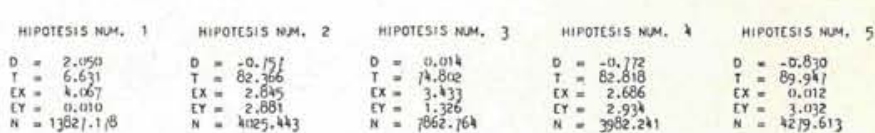

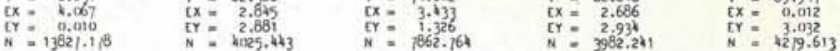

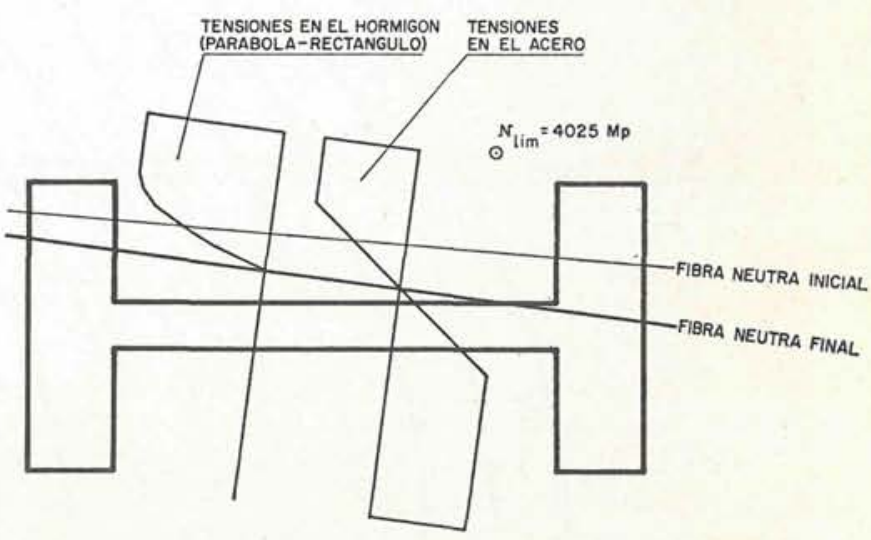

resultados (hip. 2.:)

Programa para sección de forma arbitraria. 


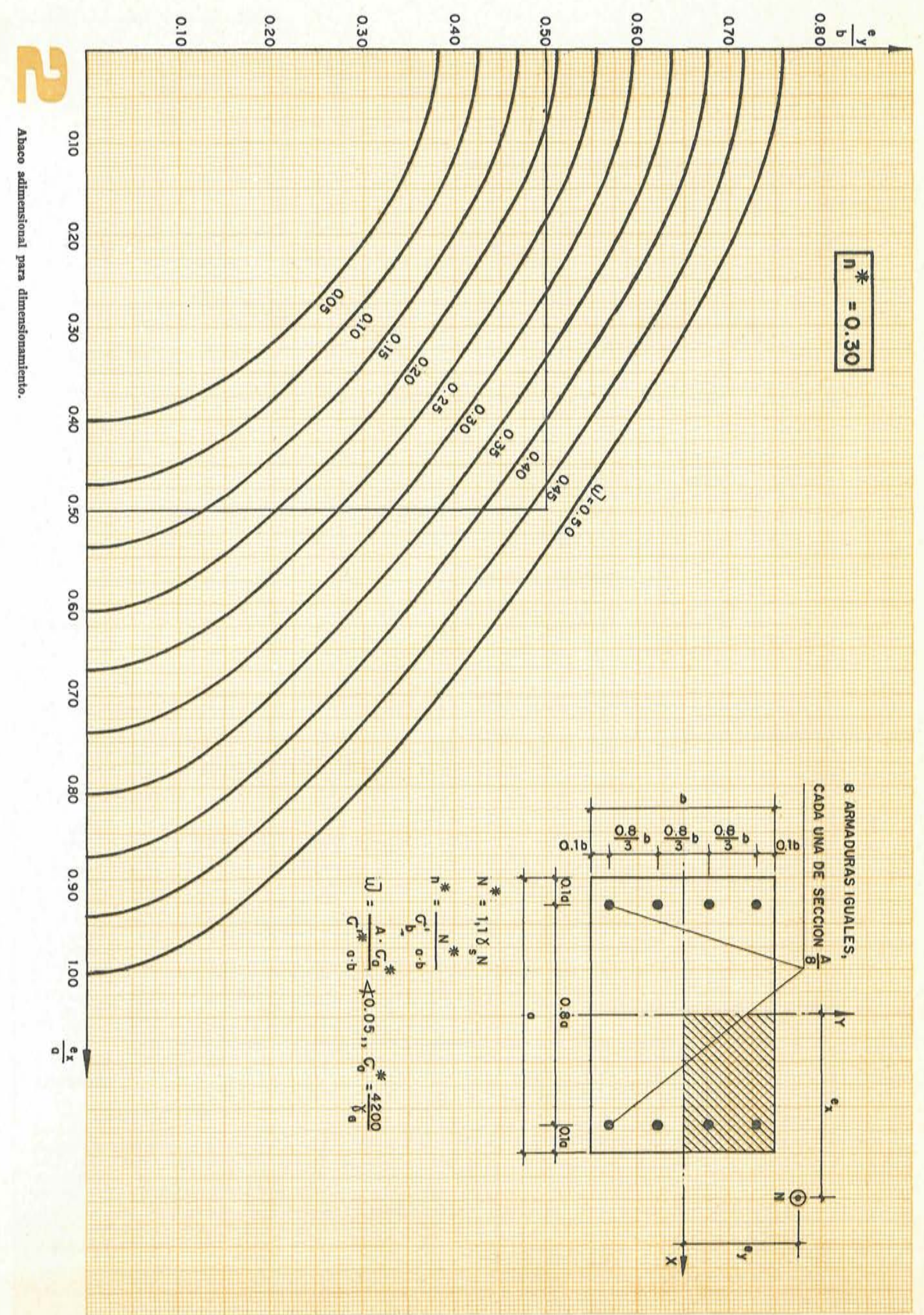


No parece ocurrir lo mismo para otras disposiciones del nomograma que fueron ensayadas, o si se cambian las variables del mismo (por ejemplo, empleando momentos reducidos en los ejes en vez de excentricidades reducidas).

El uso del nomograma es rápido y sencillo, tanto en dimensionamiento como en comprobación.

En el primer caso son datos las excentricidades y el esfuerzo axil reducido $n$. Siguiendo el camino indicado en línea gruesa con puntas de flecha en la figura 4, se llega a determinar la cuantía mecánica $\varpi$. En el segundo, en el que esta cuantía es dato, basta invertir el sentido del recorrido de la línea gruesa de la derecha para calcular el esfuerzo axil resistido $n$.

Es necesario advertir también aquí que las bases que han servido para la elaboración del nomograma (deformaciones de rotura y diagrama del hormigón) no coinciden estrictamente con las adoptadas por la Instrucción Española de Hormigón E.H. 68, por lo cual el Instituto Eduardo Torroja espera publicar en breve nomogramas actualizados para éste y otros casos.
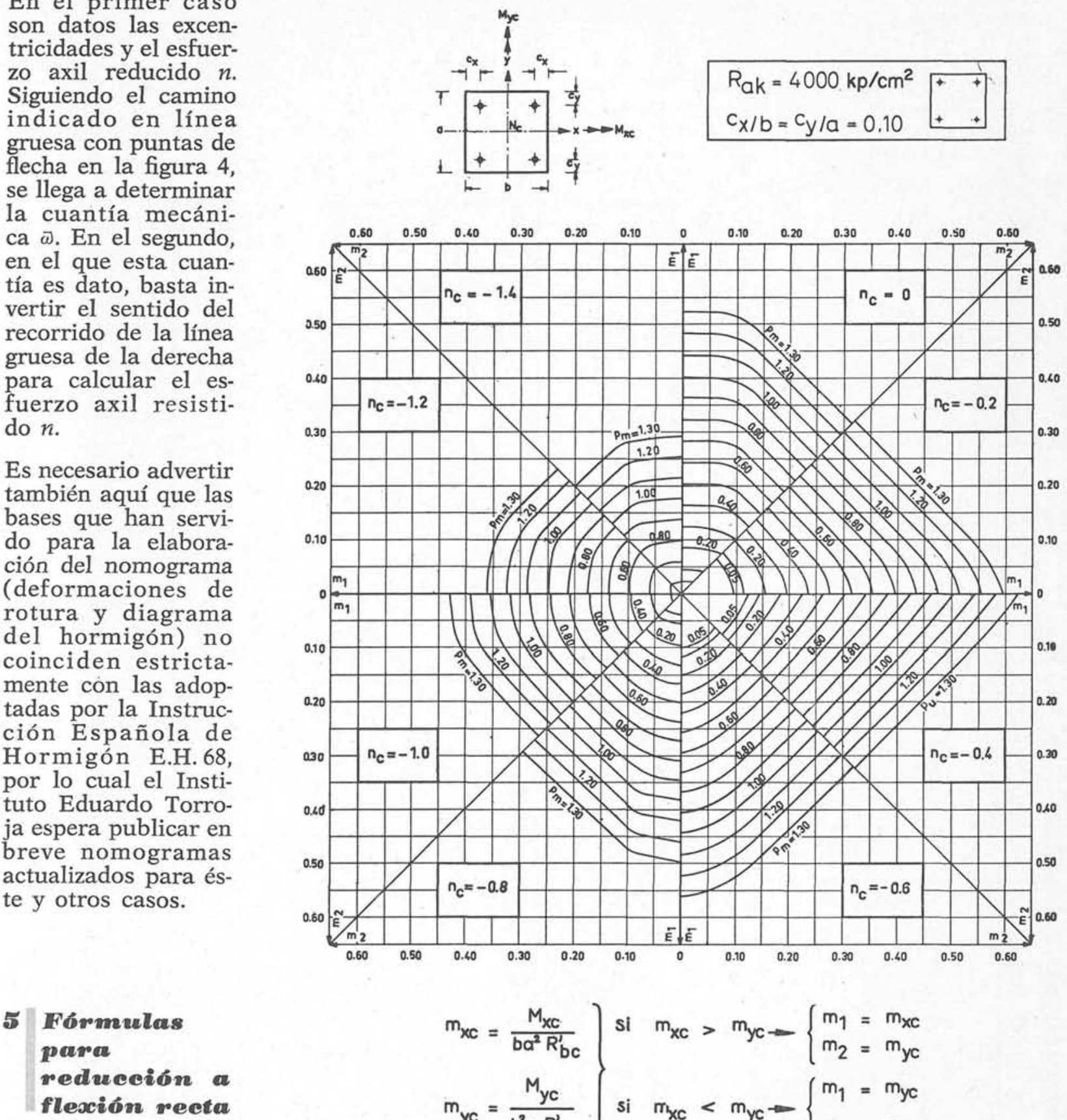

Un paso más en la simplificación del problema de la flexión esviada consiste en su reducción a flexión recta mediante alguna fórmula aproximada.

$$
\begin{aligned}
& \left.m_{x c}=\frac{M_{x c}}{b a^{2} R_{b c}^{\prime}}\right\} \text { si } m_{x c}>m_{y c}-\left\{\begin{array}{l}
m_{1}=m_{x c} \\
m_{2}=m_{y c}
\end{array}\right. \\
& \left.m_{y c}=\frac{M_{y c}}{b^{2} a R_{b c}^{\prime}}\right\} \text { si } m_{x c}<m_{y c}-\left\{\begin{array}{l}
m_{1}=m_{y c} \\
m_{2}=m_{x c}
\end{array}\right. \\
& n_{c}=\frac{N_{c}}{b a R_{b c}^{\prime}} \\
& A_{a}=P_{m} \frac{R_{b c}^{\prime}}{R_{a c}} a b=P_{m} \cdot \frac{R_{b c}^{\prime}}{R_{a k}} 1,15 \cdot a \cdot b
\end{aligned}
$$

Roseta de dimensionamiento (Tomada de un documento de trabajo de la Comisión III, "flexión-compresión" del C.E.B., preparado por E. Grasser, de Münich). 


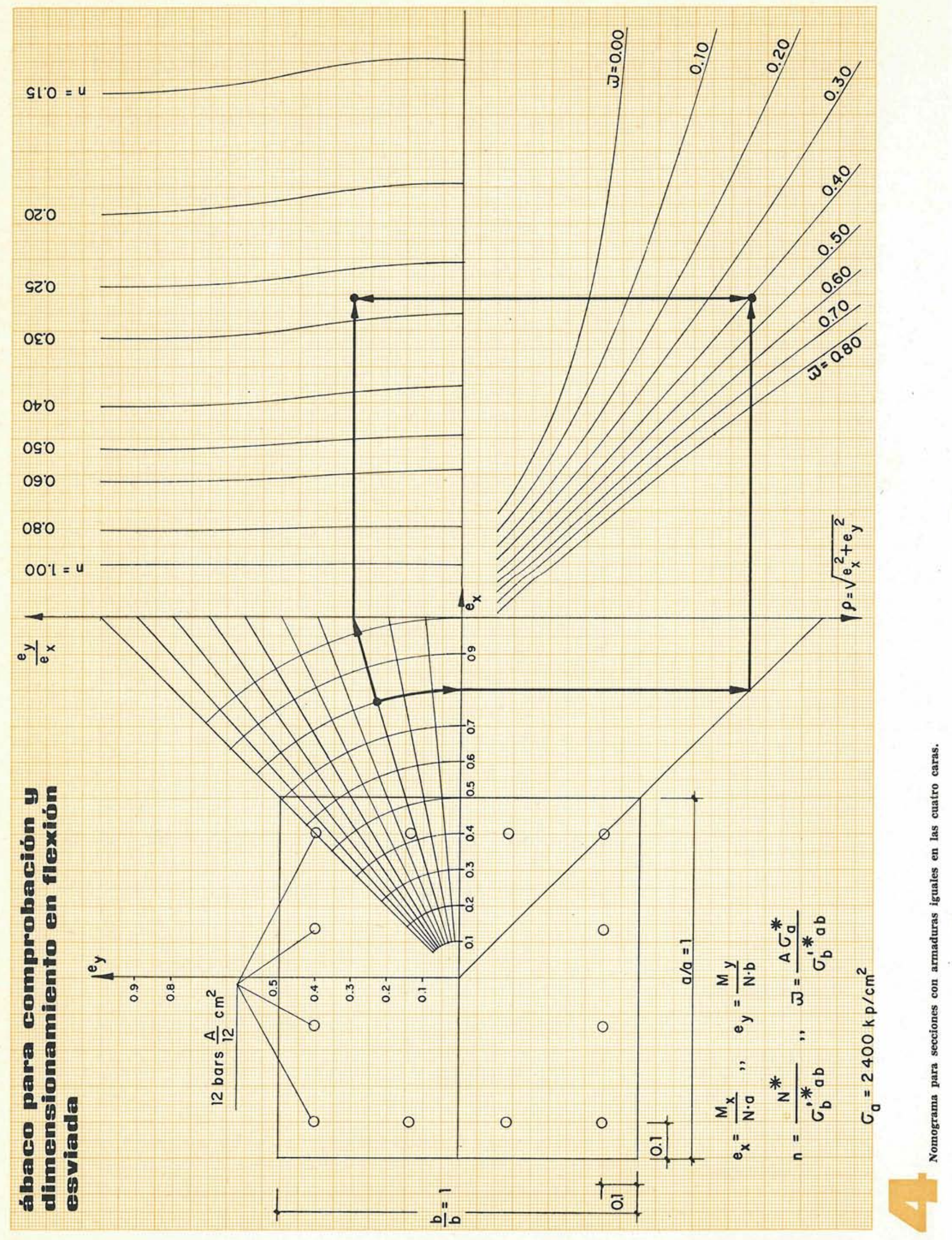




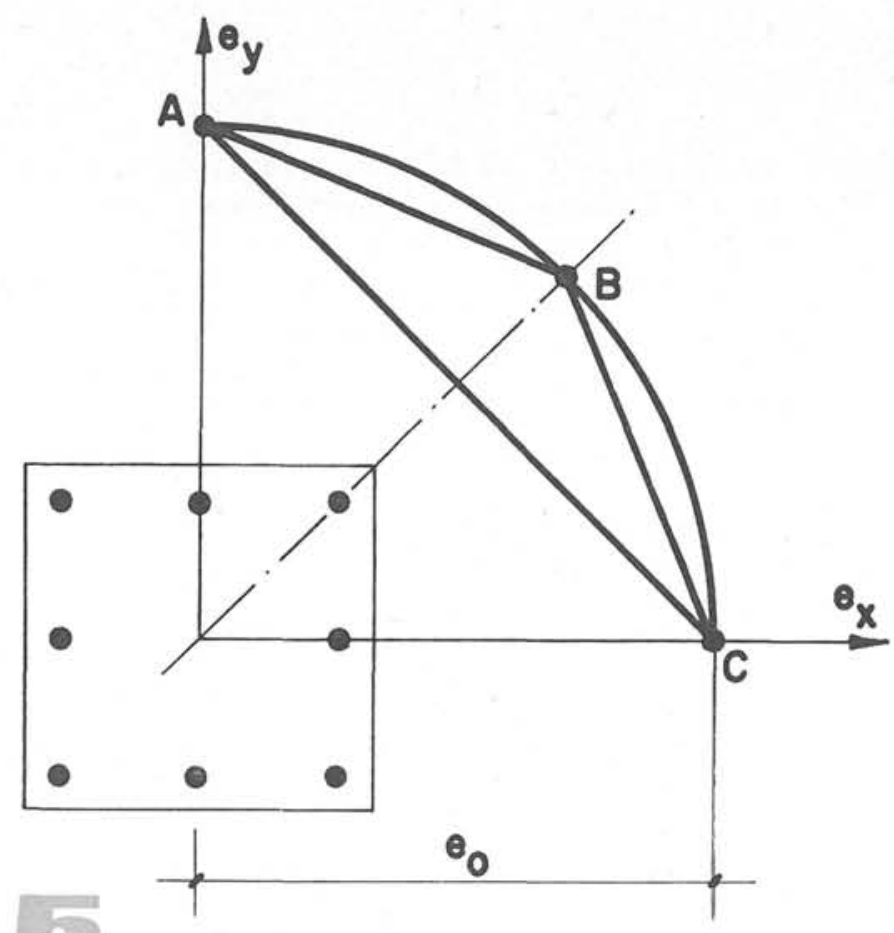

Reducción a flexión recta.

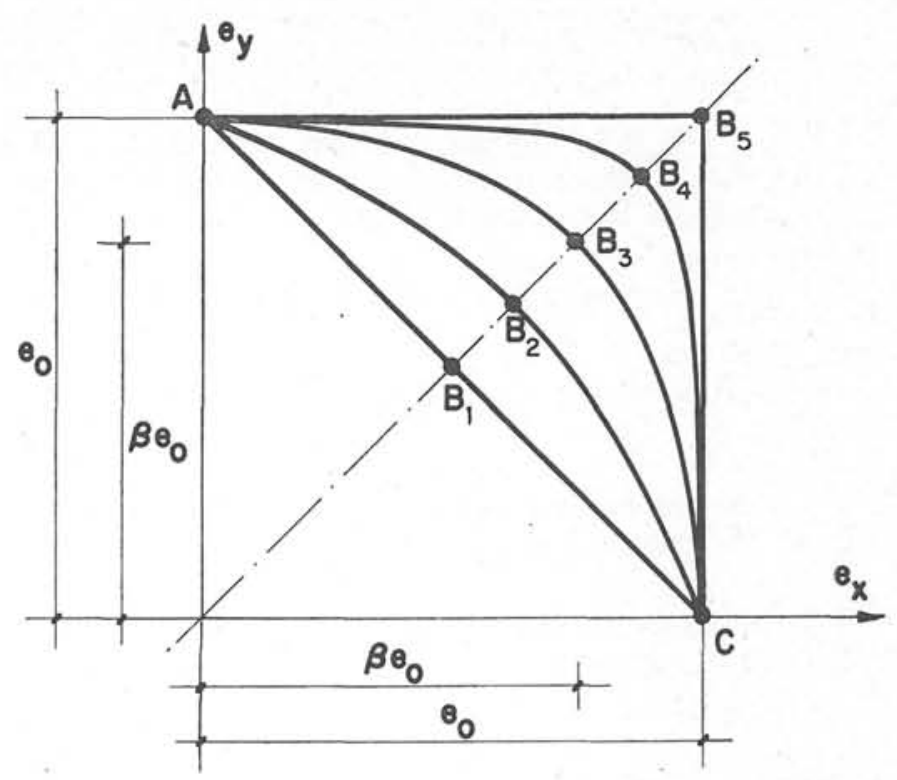

Curvas de igual resistencia.

Sea, por ejemplo, una sección cuadrada con armadura bisimétrica como la de la figura 5 (una sección rectangular puede reducirse a otra cuadrada mediante una afinidad). Sea $A B C$ la curva de igual resistencia correspondiente a un cierto axil $N$ y a una cierta cuantía $\omega$. Quiere esto decir que la sección, armada con dicha cuantía, se agota para la fuerza $N$ si ésta actúa en cualquiera de los puntos de la curva $A B C$. Por tanto, el problema de dimensionamiento de la sección sometida a fuerza $N$ actuando con doble excentricidad en cualquier punto $\left(e_{x}, e_{y}\right)$ de la curva es equivalente al del dimensionamiento para la fuerza $N$ actuando con una única excentricidad $e_{0}$ en $C$ (flexocompresión recta). Conociendo la forma de la curva es evidente que se puede calcular esta excentricidad equivalente $e_{0}$.

Ahora bien, puesto que la curva no tiene, en general, una expresión analítica sencilla, es necesario sustituirla por otra que sí la tenga, aun a costa de cierta pérdida de exactitud.

Una primera propuesta es la de A. Aas-Jakobsen (3), consistente en asimilar la curva a un círculo. De aquí resulta:

$$
e_{0}=\sqrt{e_{x}^{2}+e_{y}^{2}} .
$$

Esta expresión es muy sencilla y en muchos casos suficientemente aproximada. No obstante, del examen de las curvas de igual resistencia se deduce que su forma varía (fig. 6) muy marcadamente, llegando casi al límite $A B_{1} C$, por un lado, y casi al $A B_{5} C$, por el otro. Es fácil ver que para todos los casos en que (como sucede en el $A B_{2} C$ ) la curva es más plana que el círculo, la fórmula [1] proporciona dimensionamientos inseguros, a veces del $40 \%$ y más en cuantías. Parece, pues, conveniente introducir en la fórmula algún parámetro para tener en cuenta el mayor o menor aplanamiento de la curva.

En esta línea está el procedimiento de Parme (4), que conduce a la fórmula:

$$
e_{0}=\left(e_{x}^{m}+e_{y}{ }^{m}\right)^{1 / m},
$$

que puede considerarse una generalización de la anterior. En ella es

$$
m=-\frac{\log 2}{\log \beta}
$$


y $\beta$ la relación entre la excentricidad en $B$ y la excentricidad en $C$, para la curva correspondiente. Para $\beta=0,5$ se tiene $m=1$ y resulta la recta $A B_{1} C$; para $\beta=1$ se tiene $m=\infty$ y resulta la quebrada $A B_{5} C$.

En la misma línea está la fórmula de los autores:

$$
e_{0}=\frac{e_{x}+e_{y}}{2}+\sqrt{\left(\frac{e_{x}+e_{y}}{2}\right)^{-}-m^{\prime} e_{x} e_{y}}, \quad \text { con } \quad m^{\prime}=\frac{2 \beta-1}{\beta^{2}}
$$

con el mismo significado de $\beta$ y los mismos límites que en la de Parme, pero quizá de más fácil aplicación, al adoptar una forma cuadrática en vez de exponencial.

Otra posibilidad es la adoptada por la Instrucción E.H. 68, a propuesta del profesor Jiménez Montoya (5):

$$
e_{0}=e_{x}+k e_{y} \quad \text { para } \quad e_{x}>e_{y}, \quad \text { con } \quad k=\frac{1-\beta}{\beta}
$$

que tiene la ventaja de su gran sencillez, por una parte, y de quedar siempre del lado de la seguridad, por otra. En efecto, el empleo de esta fórmula equivale a la sustitución de la curva $A B C$ (fig. 5) por las cuerdas $A B$ y $B C$, que son siempre interiores a ella. La pérdida en exactitud del ajuste queda compensada por estas ventajas.

Para aplicar cualquiera de estas fórmulas es necesario el conocimiento previo del valor del parámetro correspondiente, $m, m^{\prime}$ o $k$, que, como se ha visto, es una función de $\beta$, relación del momento resistido en flexión diagonal al momento resistido en flexión recta. Estos parámetros dependen de los siguientes factores:

1. Disposición de las armaduras.

2. Recubrimiento.

3. Límite elástico del acero, $\sigma_{e}$.

4. Cuantía, $\varpi$.

5. Esfuerzo axil reducido, $n$ (también llamado $v$ ).

Deben, por tanto, suministrarse al proyectista tablas o diagramas que recojan los valores de $k$ a emplear, pudiéndose eventualmente eliminar alguno de estos factores, siempre que los valores dados sean los más desfavorables posibles para cualquier valor de los factores eliminados. Así, la Instrucción Española da unos valores en función de los dos últimos, y en las figuras 7,8 y 9 se dan gráficos en los que se tiene en cuenta además el tercero (de nuevo cabe advertir que las bases que han servido para su obtención no coinciden con las de la E.H. 68).

Por último, existe la posibilidad de simplificar aún más empleando la fórmula:

$$
e_{0}=e_{x}+e_{y}
$$

que también está siempre del lado de la seguridad, puesto que equivale a la sustitución de la curva $A B C$ (fig. 5) por la cuerda $A C$. Su principal ventaja es la de no emplear ningún parámetro auxiliar; la pérdida de exactitud es, en general, más elevada que en las fórmulas anteriores, todo lo cual hace que esta fórmula sea muy adecuada para su uso en tanteos y predimensionamiento.

En estas fórmulas se ha considerado la reducción a flexión recta en el caso de sección cuadrada con armadura bisimétrica, para simplificar la exposición. No obstante, es también posible resolver el problema en el caso más general de sección rectangular con armadura cualquiera. En esta línea, sin embargo, no se ha llegado aún a resultados que pudieran considerarse definitivos. 

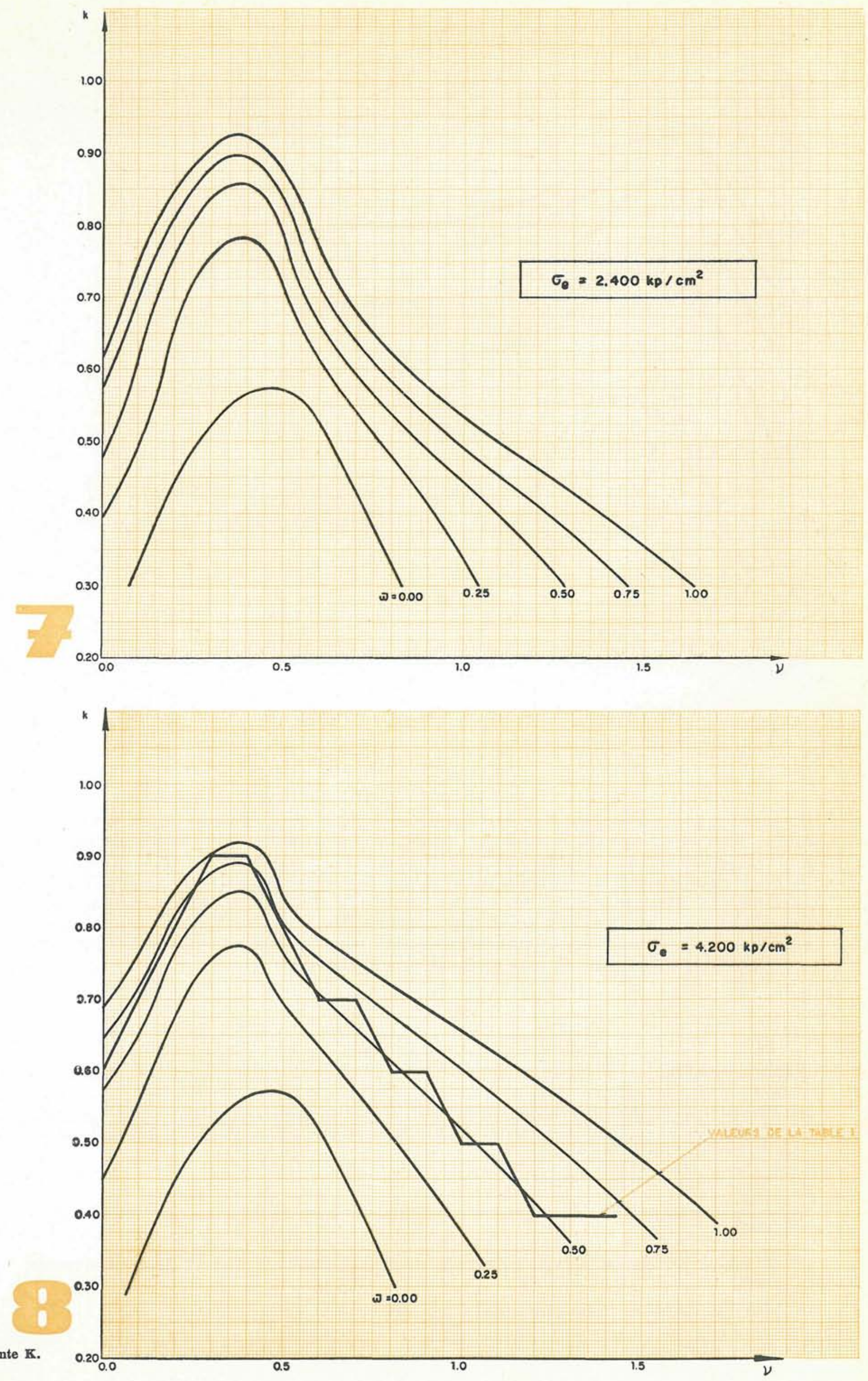

Valores del coeficiente $\mathbf{K}$. 


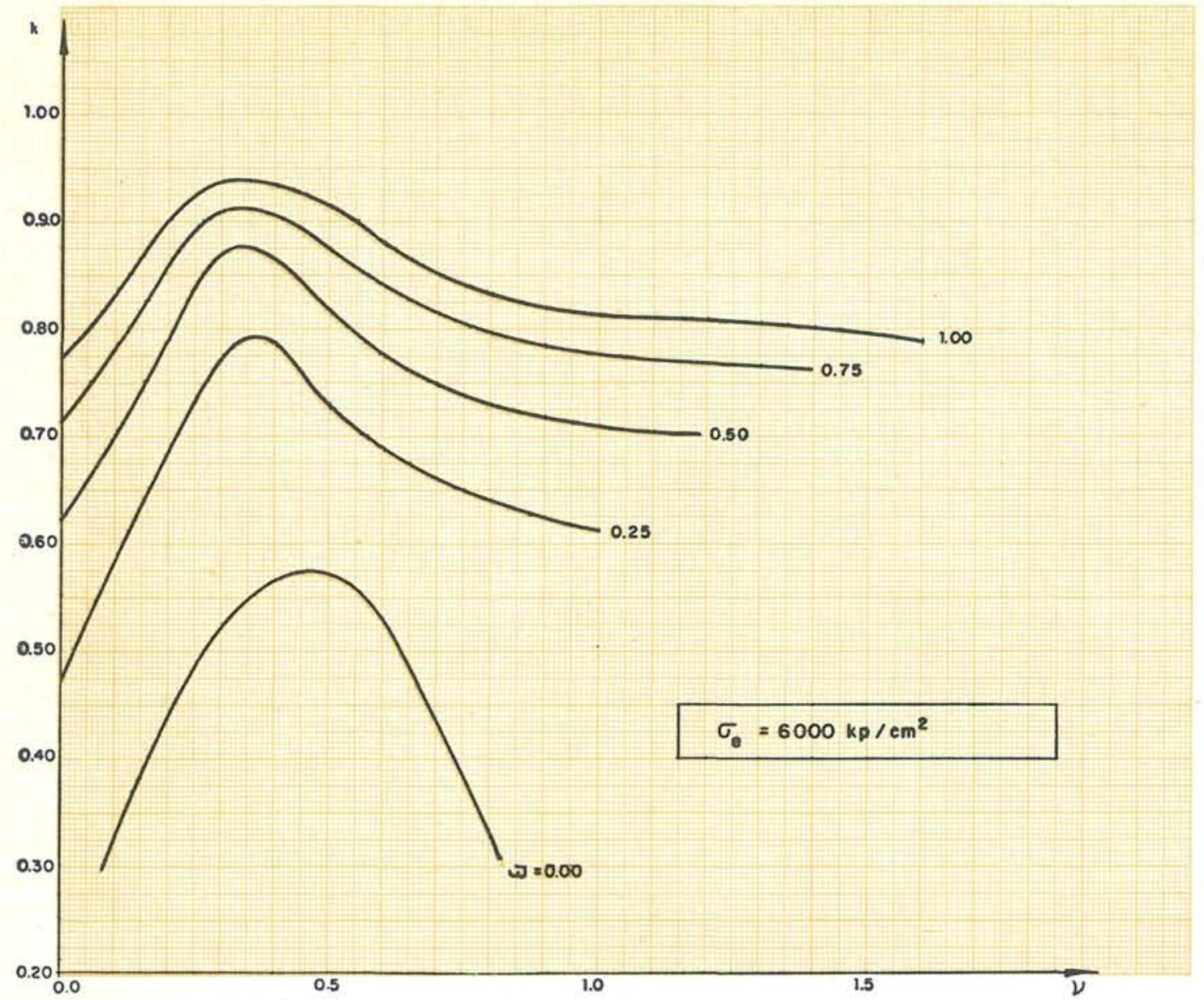

Valores del coeficiente $K$.

Hasta aquí se ha supuesto que las hipótesis básicas de la flexión recta eran aplicables al caso de flexión esviada sin ninguna modificación. La justificación de esta generalización no es evidente, particularmente en lo que respecta al diagrama tensión-deformación del hormigón y a los estados de deformación que caracterizan el agotamiento. Sólo mediante un análisis detallado de los resultados experimentales existentes, y de los que se obtengan en el futuro, podrá llegarse a un conocimiento más profundo del comportamiento de una sección de hormigón armado solicitada en flexión, en el más general de los casos.

\section{Bibliogreafía}

(1) Moran, Garcfa Meseguer, Martínez Calzón y Nadal: Estudio teórico-experimental de la flexocompresión esviada de secciones de hormigón armado. Monografía del I.E.T.c.c., número $265-266$ (septiembre 1967).

(2) Instrucción española E.H. 68.

(3) A. AAS-JAKOBSEN: "Biaxial eccentricities in ultimate load design», Journal A.C.I. (marzo 1964).

(4) PARME: «Rectangular Columns Subjected to biaxial bending». Journal A.C.I. (septiembre 1966).

(5) Jiménez MonTOYA: Hormigón armado, pág. 245. 


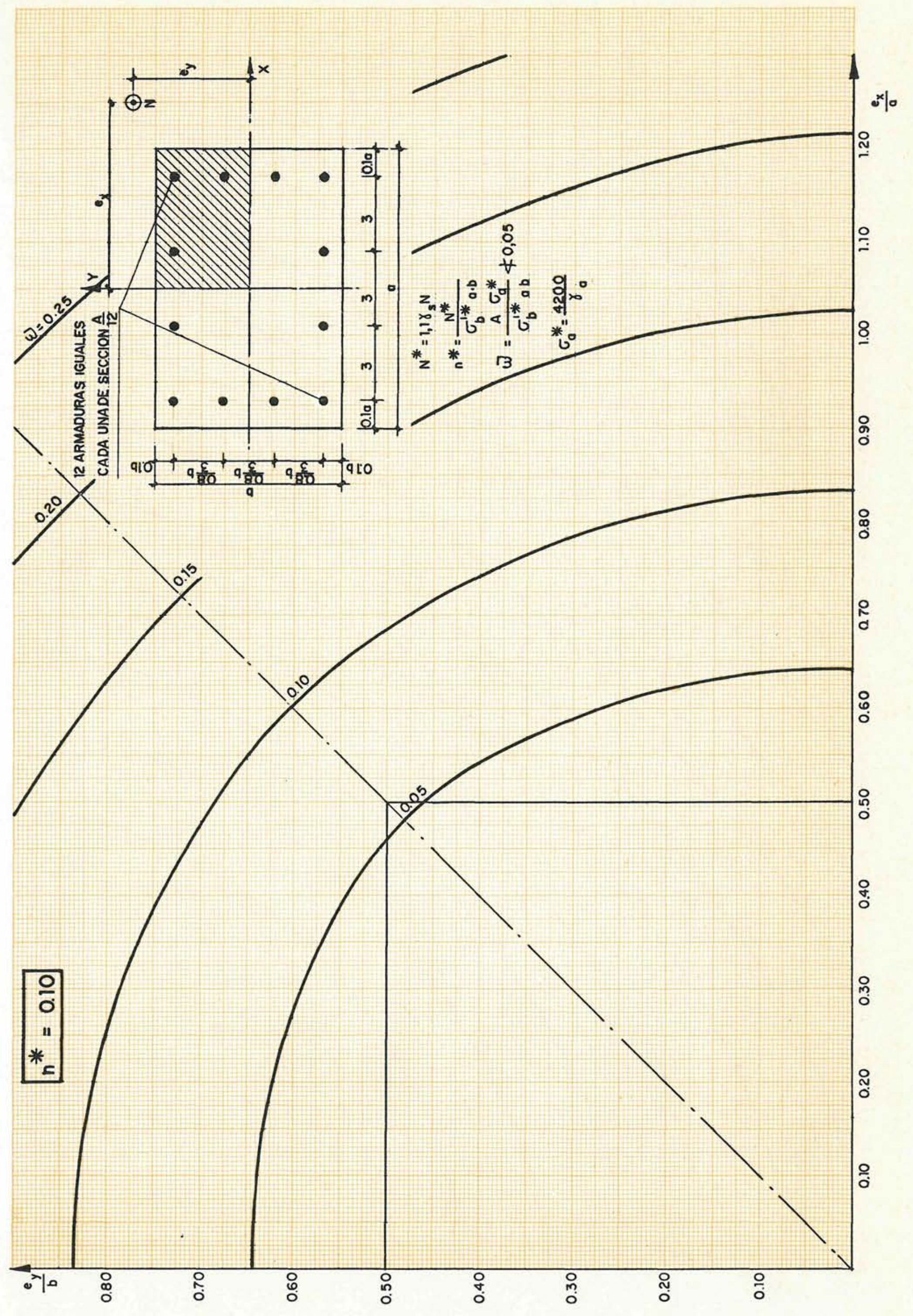




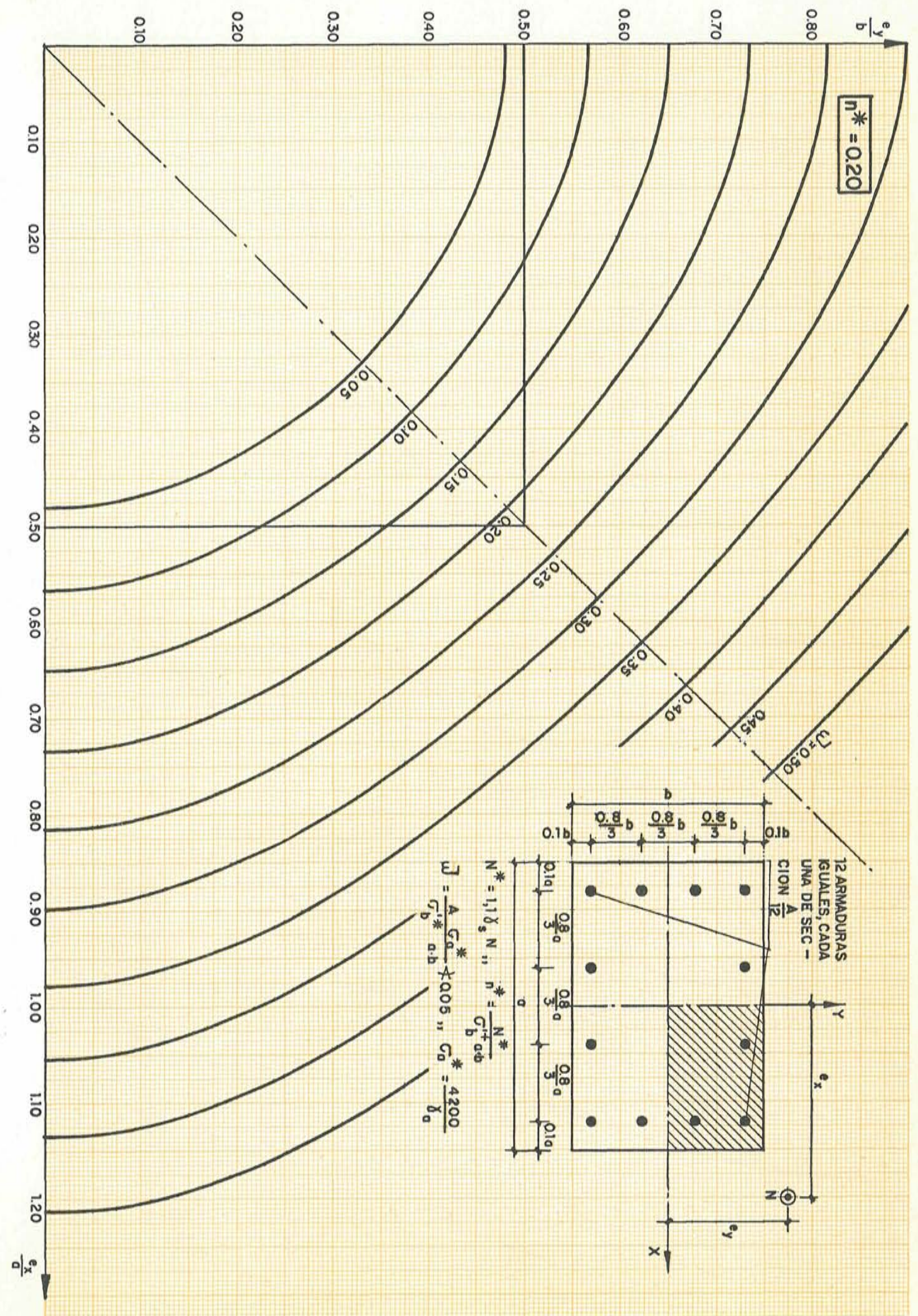




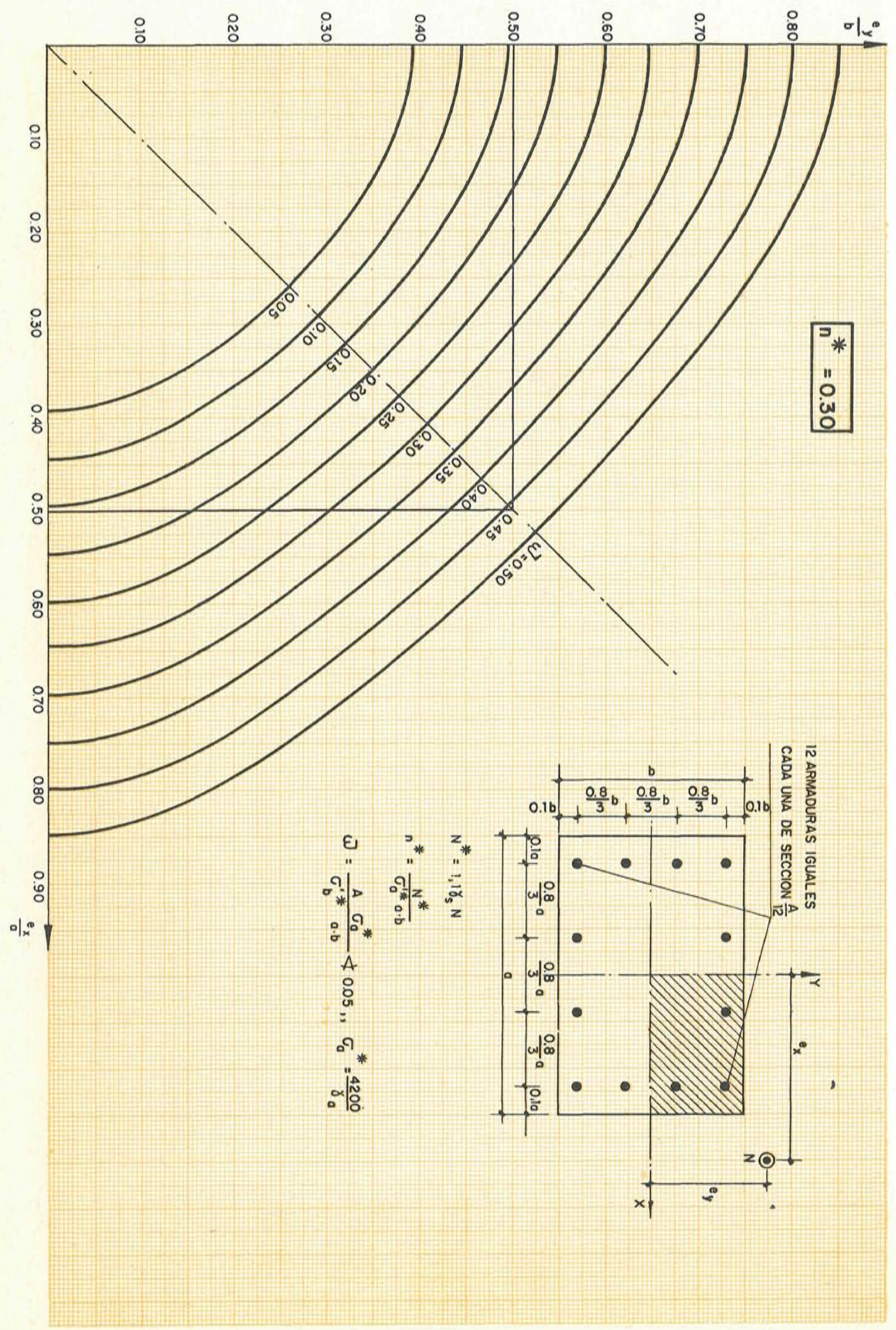




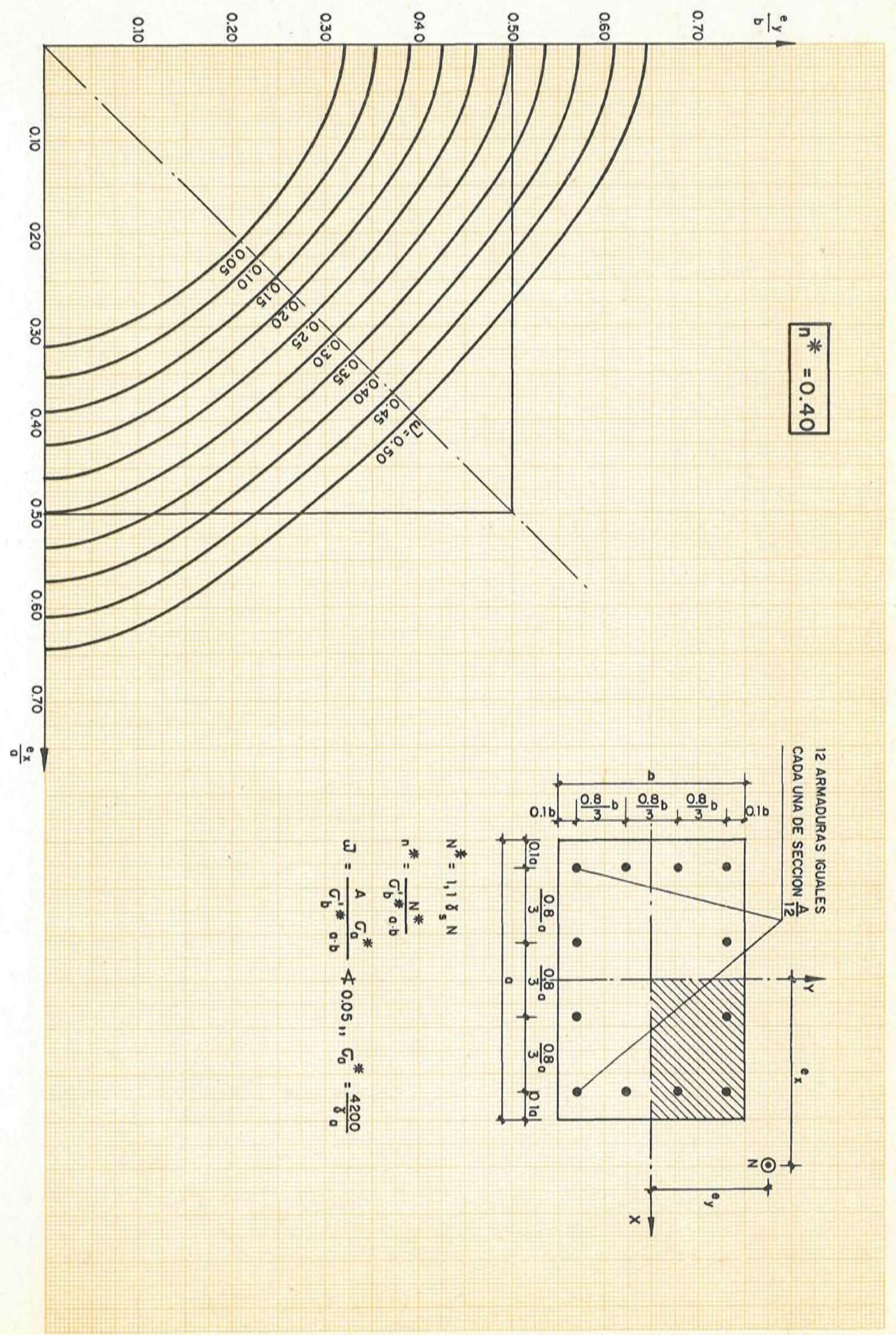



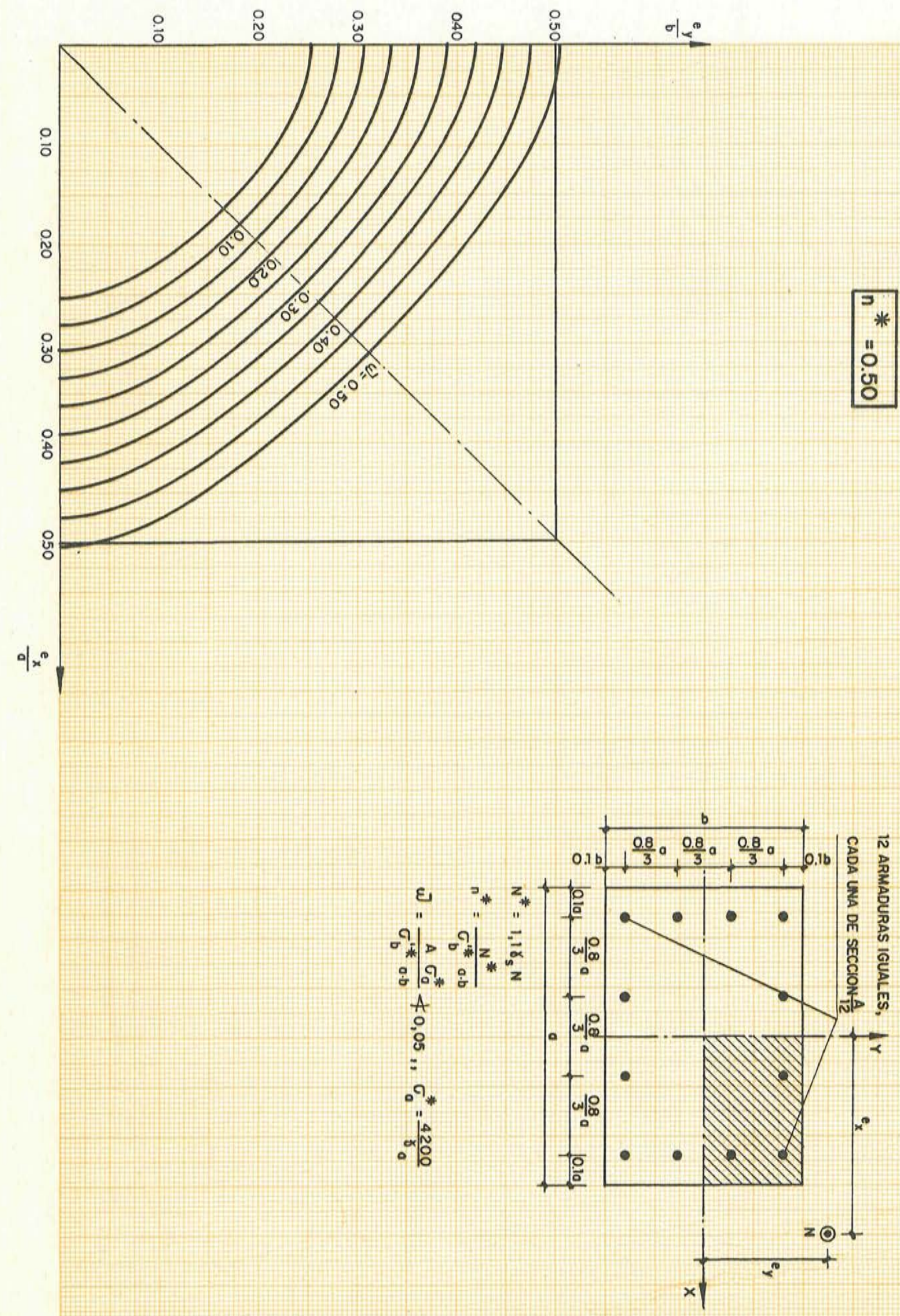


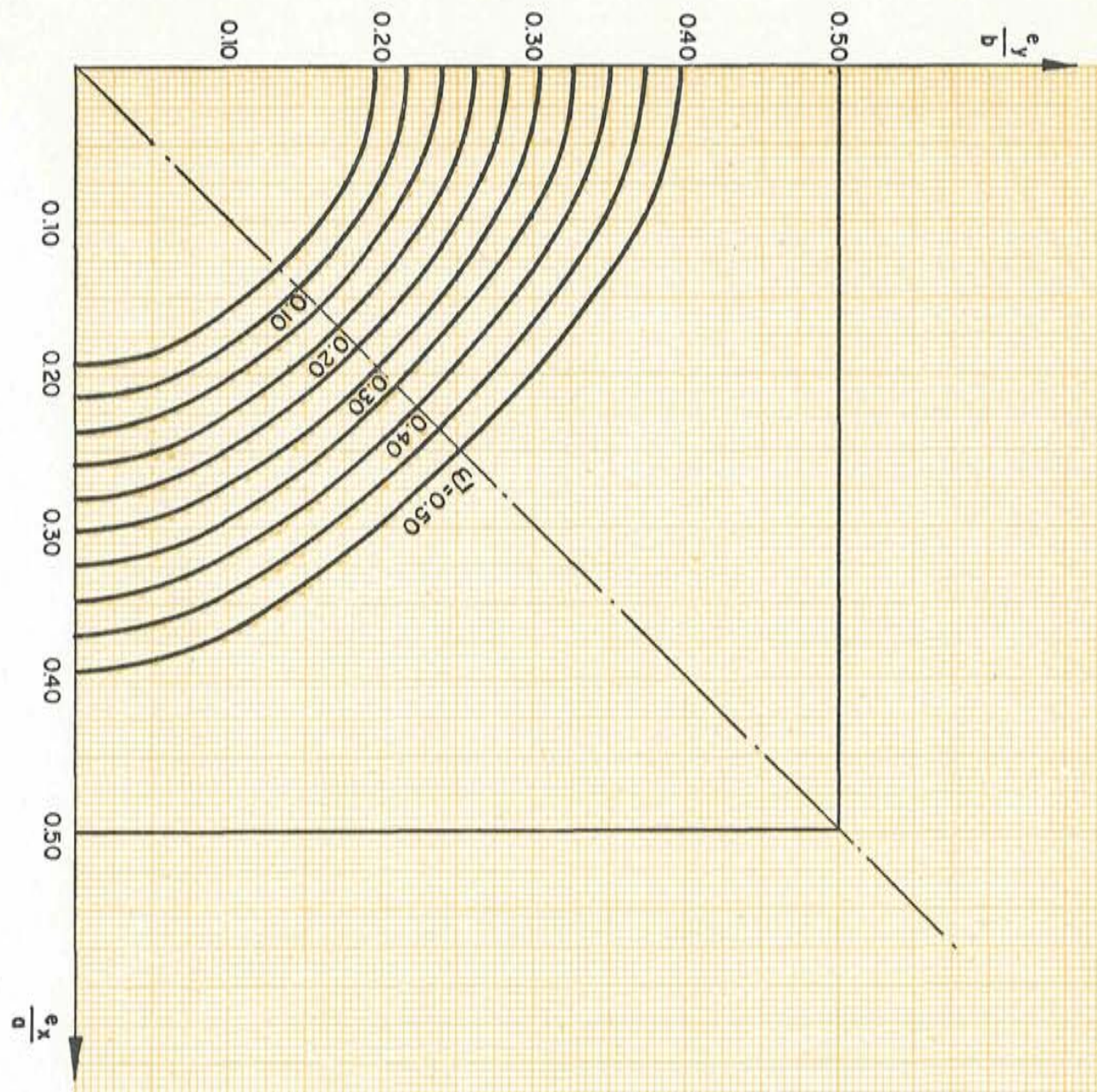

\begin{tabular}{|c|}
\hline 米 \\
11 \\
0 \\
0 \\
0 \\
\hline
\end{tabular}

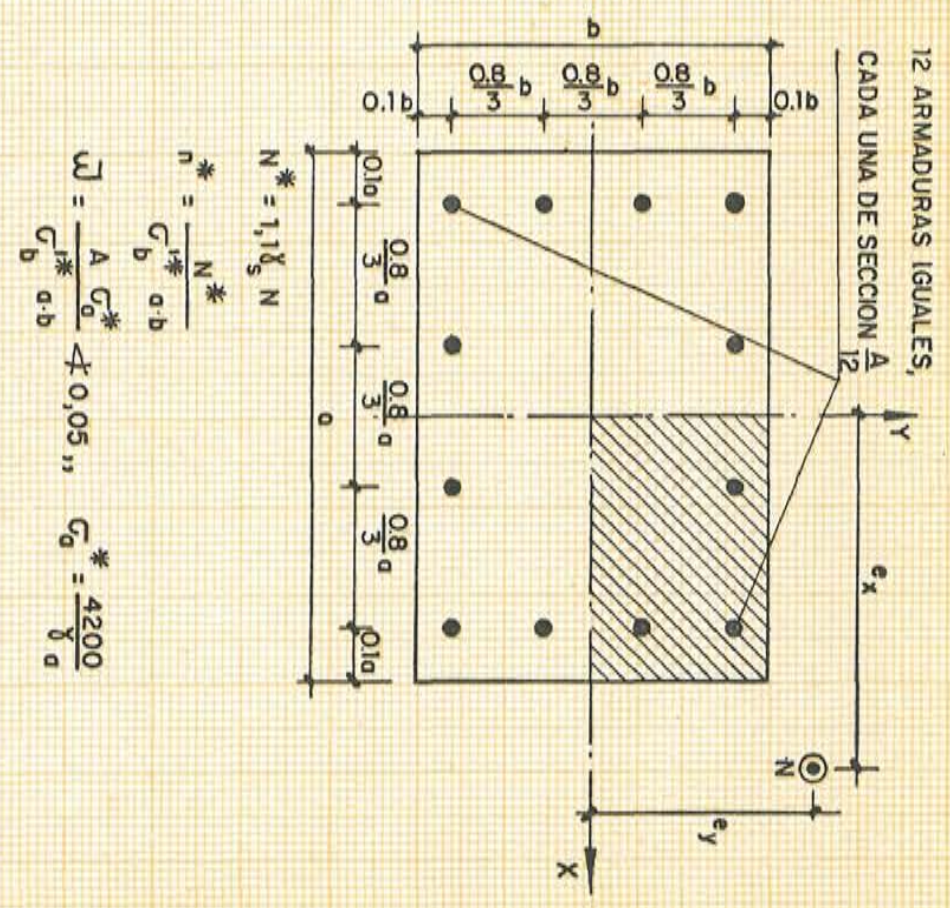



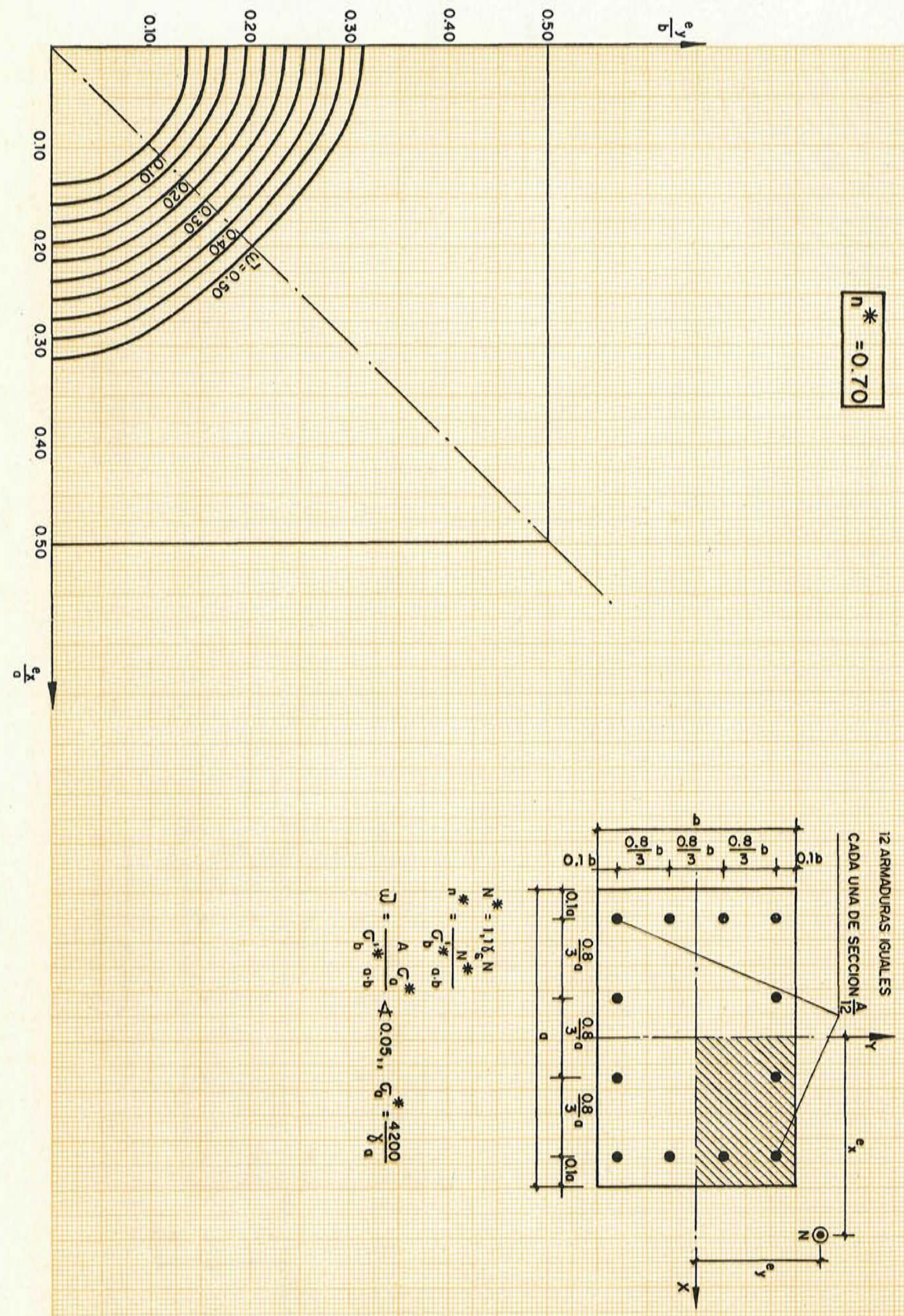


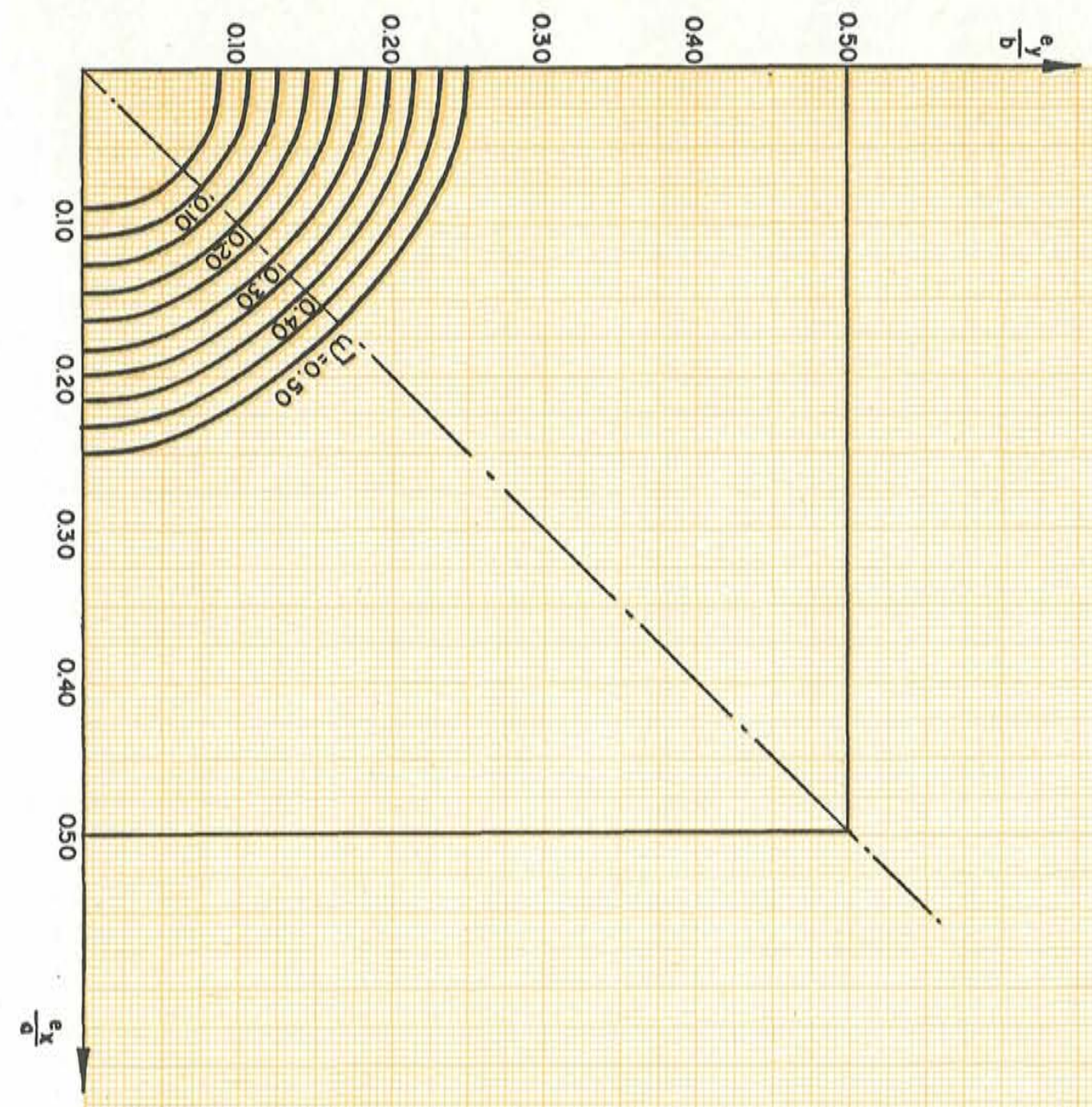

\begin{tabular}{c}
$7_{*}$ \\
11 \\
0 \\
0 \\
0 \\
\hline
\end{tabular}

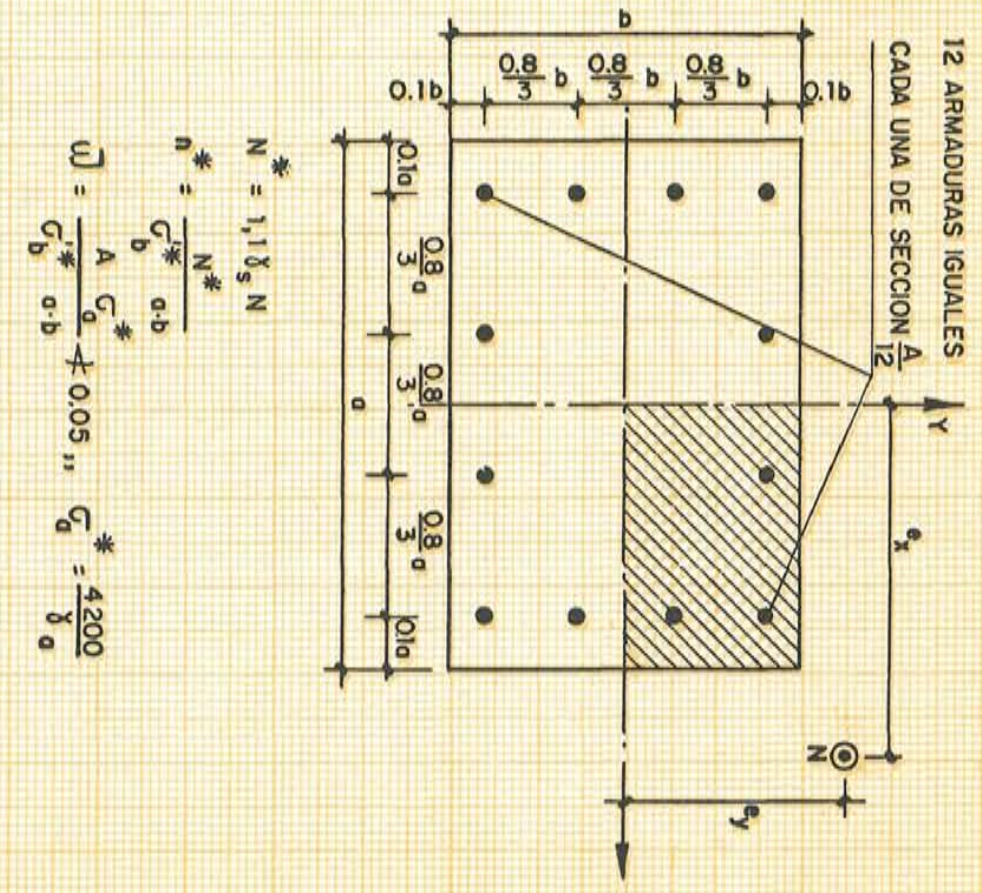




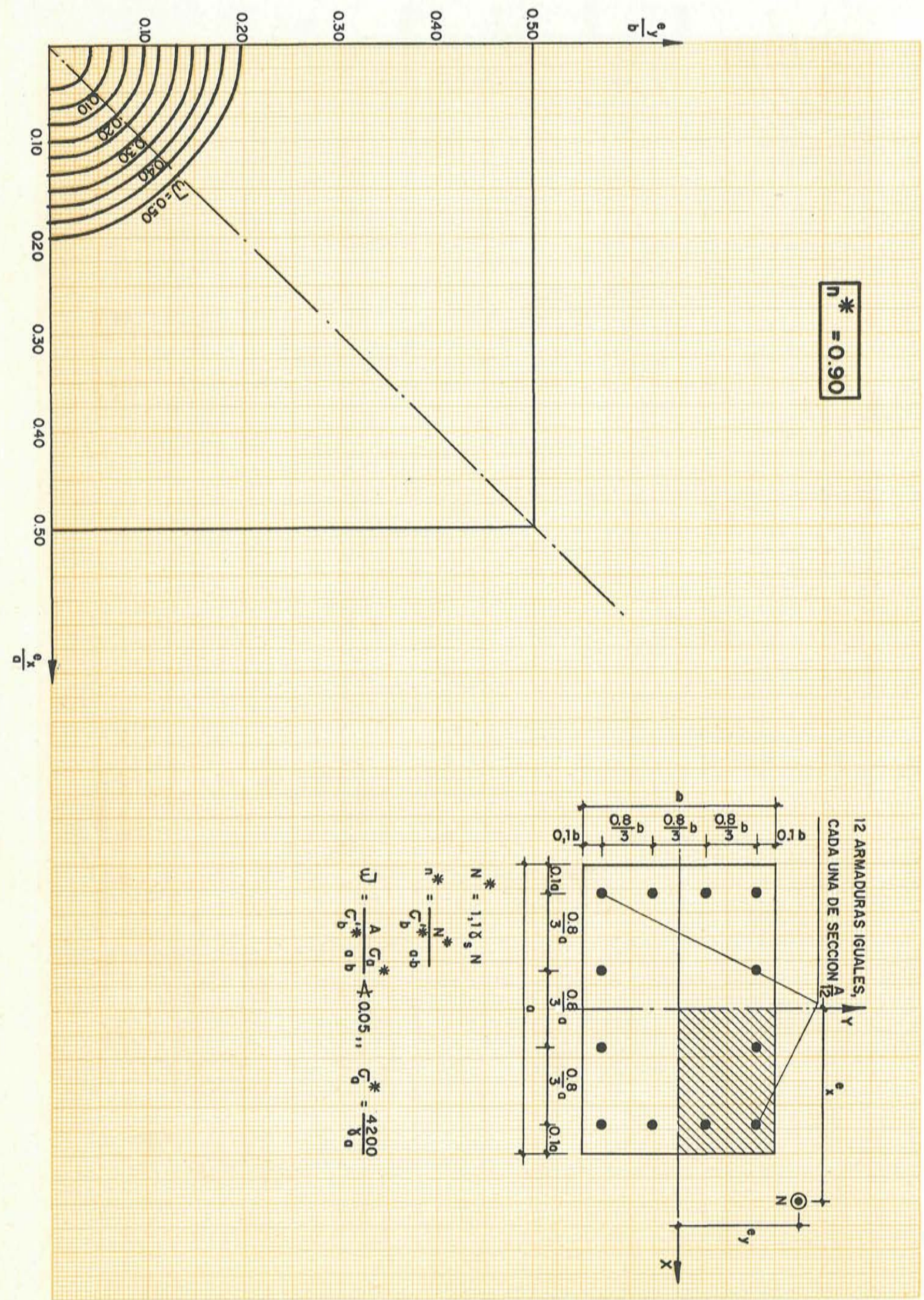




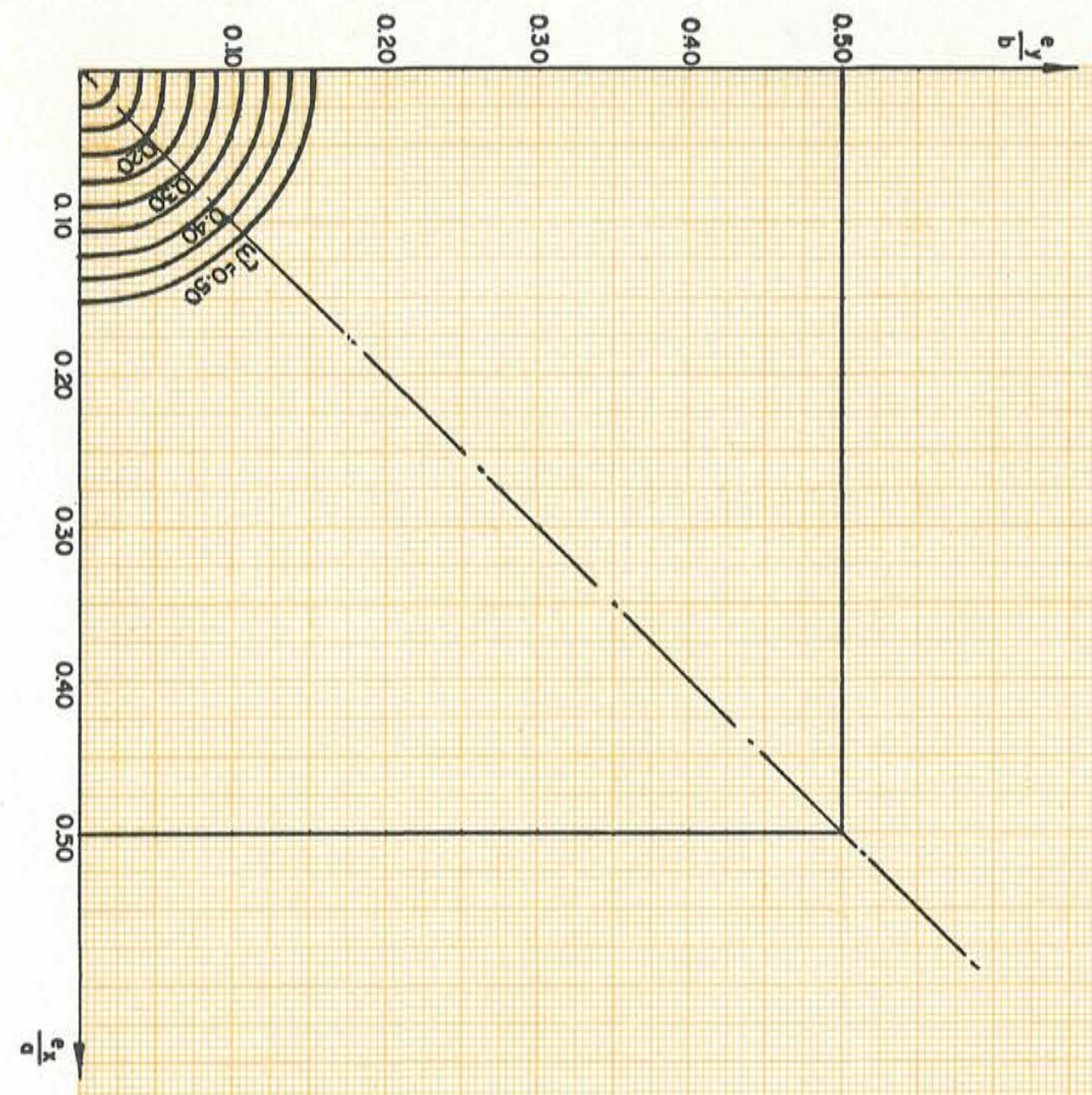

廉

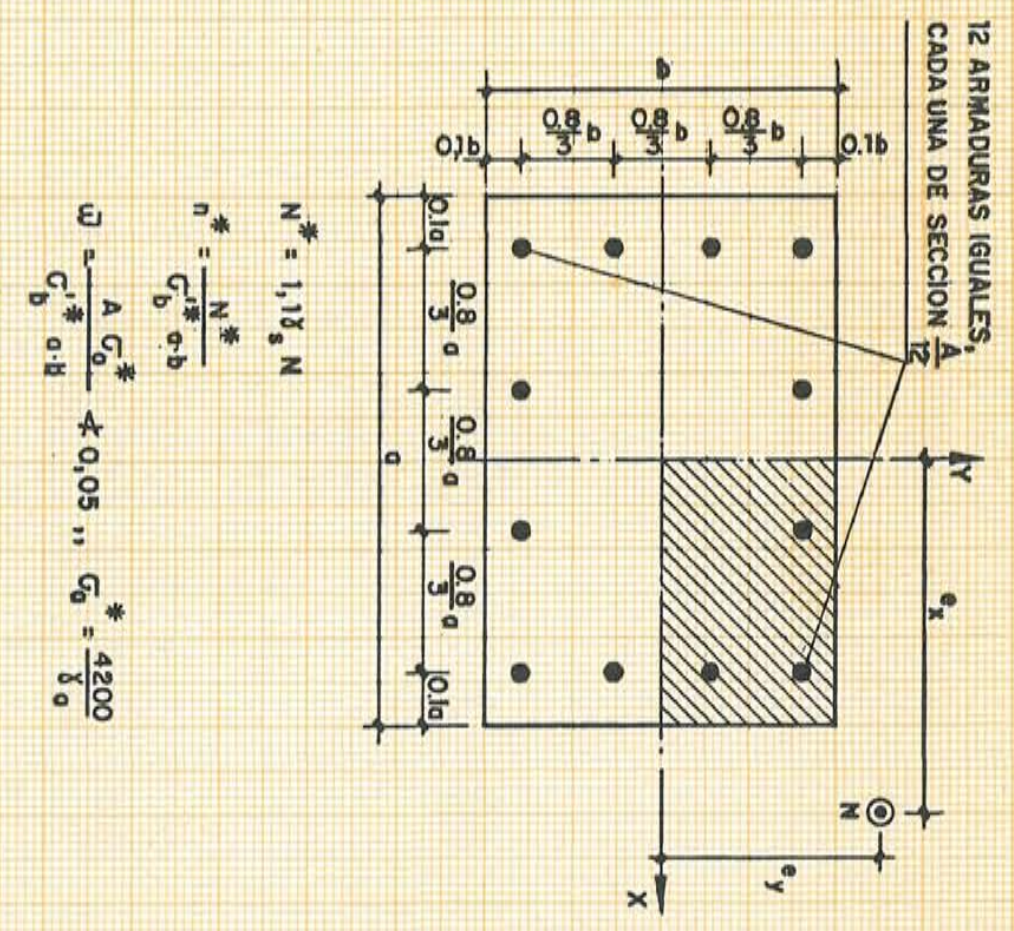




\section{Flexion díviée: état actuel}

F. Morán et A. Verde, ingénieurs des Ponts et Chaussées

Les auteurs exposent brièvement les différentes possibilités d'effectuer le dimensionnement pratique des sections en béton armé, sollicitées en flexocompression déviée, dans le domaine du calcul en rupture.

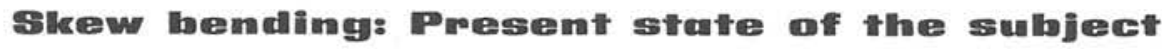

F. Morán \& A. Verde, civil engineers

A brief report is made of present available methods for the practical design calculation of reinforced concrete sections subjected to skew bending with compression, assuming methods of calculation based on ultimate strength capacity.

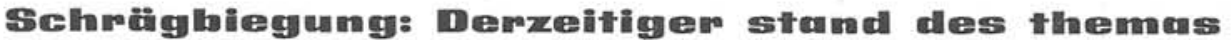

F. Morán und A. Verde, Tiefbauingenieure

Es werden hier kurz die verschiedenen bestehenden Möglichkeiten dargestellt einer praktischen Dimensionierung dr Eisenbetonqurschnitte, die erforderlich sind bei schräger Biegekompression, in Rahmen der Bruchberechnungen. 